\title{
Anticipo del futuro o ritorno al passato?
}

\author{
Elio Borgonovi
}

Il 28 luglio, con il quarantaduesimo voto di fiducia, il Senato ha approvato il Decreto sugli enti locali che comprende norme di contenimento della spesa del SSN per 2,35 miliardi. A seguito di questo voto è iniziato il solito gioco delle parti poiché molte Regioni hanno contestato la misura e accusato il Governo di attuare un ulteriore taglio della spesa per la tutela della salute. Posizione delle Regioni capitanata soprattutto dal Veneto per voce del Governatore Luca Zaia e dell'Assessore Luca Coletto e dalla Lombardia per voce del Governatore Roberto Maroni, al quale è stata attribuita dalla stampa l'affermazione à la guerre comme à la guerre. Immediatamente il Governo, per voce dei Ministri Maria Elena Boschi e Beatrice Lorenzin si è affrettato ad affermare che si trattava della semplice applicazione dell'accordo Stato-Regioni raggiunto il 2 luglio. In effetti entrambe le parti erano portatrici di mezze verità in quanto è vero che il 2 luglio era stato concretizzato l'accordo per distribuire tra le Regioni il minor trasferimento di 4 miliardi (di cui 2,35 imputabili al FSN e 1,65 per gli altri settori di intervento) già previsto nella Legge di Stabilità per il 2015, ma è anche vero che «molte Regioni potevano dichiarare che non si è tenuto conto dei costi standarde delle differenziazioni delle Regioni cosiddette virtuose e Regioni meno virtuose».

Il balletto si è puntualmente ripetuto appena presentata la proposta del Governo di Legge di Stabilità per il 2016, che prevede per il FSN uno stanziamento di 111 miliardi. Le Regioni sono scese sul piede di guerra affermando che si è in presenza di un ulteriore taglio del finanziamento al SSN, mentre il Primo Ministro Renzi, sceso in campo direttamente, ha sfidato le Regioni sul piano delle cifre, affermando che si trattava comunque di un aumento rispetto al finanziamento per l'anno precedente. Preceduta da una alquanto discutibile affermazione attribuita dai media al Primo Ministro, secondo cui nell' incontro con le Regioni programmato per il 4 di novembre «ci divertiremo», il Governo ha lanciato il guanto di sfida alle Regioni per il recupero di efficienza, di lotta agli sprechi e alla corruzione. In effetti il tema è tremendamente serio per la qualità di vita di 60 milioni di italiani (la tutela della salute è un diritto della persona) per poterlo liquidare con una battuta a effetto e con una eccessiva semplificazione rispetto al mondo reale. La politica del Governo si basa in sostanza su due pilastri: sul piano contabile e finanziario un aumento, seppur limitato, dello stanziamento per il FSN rispetto al 2015, sul piano sostanziale la rinnovata pressione nei confronti delle Regioni per indurle/costringerle a eliminare sprechi e inefficienze senza ricorrere al facile escamotage di rivalersi nei confronti dei cittadini tramite un aumento delle addizionali finalizzate agli interventi nel settore e dei ticket. Politica del Governo che non è infondata, in quanto nessuno può negare l'esistenza di sprechi, inefficienze, prestazioni inappropriate, fatti di corruzione, ma che deve pur essere sottoposta al vaglio di credibilità e realisticità.

Innanzitutto sul piano contabile è bene precisare che, il Patto della Salute stipulato il 10 luglio 2014, prevedeva che «il livello del finanziamento del Servizio Sanitario Nazionale a cui concorre lo Stato è previsto in 109,928 miliardi per l'anno 2014, 112,062 miliardi per l'anno 2015 e 115,444 miliardi per l'anno 2016» (articolo 1). Quindi è vero che per il 2016, 111 miliardi equivalgono a 1 miliardo aggiuntivo rispetto alla somma stanziata per il 2015 mentre è difficile negare che rispetto al totale previsto dal Patto per il triennio 2014-2016 mancano 2,35 miliardi per il 2015 e ben 4,444 per il 2016, in totale 6,794. Se poi si tiene conto che la Legge di stabilità prevede che nel fondo di 111 
miliardi per il 2016 è inclusa anche una somma di 0,800 miliardi destinata alla revisione dei LEA, si può dire che rispetto al Patto Stato-Regioni del luglio 2014 vi è un minor finanziamento per il triennio di 7,594 miliardi. È altrettanto vero che nel Patto del luglio 2014 era scritto «salvo eventuali modifiche che si rendessero necessarie in relazione al conseguimento degli obiettivi di finanzia pubblica e a variazioni del quadro macroeconomico», una specie di clausola di salvaguardia. Tuttavia, nessuno può negare che rispetto al luglio 2014 il quadro macroeconomico è nettamente migliorato per il 2015 (previsioni di aumento del PIL attorno allo 0,8-1\%) e per il 2016 (previsioni di aumento del PIL che si collocano tra 1,2 e 1,5\%). Per completezza di analisi si ricorda che la spesa pubblica contabilizzata a consuntivo per il 2013 è stata pari a 112,614 miliardi (in diminuzione di 1,2\% rispetto alla spesa del 2012), al cui finanziamento hanno contribuito 3,156 miliardi di entrate proprie varie delle Regioni (Rapporto OASI 2014 pp. 150 e 152) e che da un recente rapporto OCSE l'aumento di spesa tra il 2011 e il 2014 è stato di 1,4\% in Italia e 3,6\% nella media dei Paesi considerati.

Pur essendo chi scrive in possesso di un diploma di Ragioneria, è opportuno superare le mere questioni contabili e approfondire il dibattito sui contenuti sostanziali per capire se si è in presenza di un «anticipo del futuro», interventi per attuare finalmente una razionalizzazione della spesa o di un «ritorno al passato», perpetuazione di un sistema deresponsabilizzato a tutti i livelli. Da alcuni anni si susseguono analisi e ricerche tramite le quali si stimano $i$ «risparmi» possibili nel SSN senza che ciò debba comportare una riduzione dei livelli quali-quantitativi delle prestazioni e dei servizi garantiti ai cittadini (i LEA). Si ricorda uno studio del Commissario alla spending review Carlo Cottarelli che ipotizzava una riduzione fino al 5,7\% della spesa pubblica per la salute rispetto al 7,2\% del 2013 e a circa il 7\% attuale. Tra luglio e novembre la stampa ha fatto trapelare stime degli attuali Commissari alla spending review di possibili risparmi di 10 miliardi. Poiché tale stima, se riferita a un anno, è oggettivamente irrealistica anche per $i$ più ottimisti fautori della razionalizzazione e della lotta agli sprechi, in sede di replica al Senato a luglio il Ministro Lorenzin si è affrettata a chiarire che la stima era riferita al periodo 2015-2019 e che comunque il decreto prevedeva un minor finanziamento rispetto alle cifre previste nel Patto per la Salute del 2014 di circa 2,5 miliardi annui per il periodo 2015-16-17. Anche il dibattito (che sembra semantico ma che è di sostanza) relativo al fatto che si tratti di «tagli reali di spesa» o di «minore finanziamento rispetto alla dinamica tendenziale della spesa a legislazione invariata» lascia il tempo che trova, come si suol dire in gergo popolare. Infatti si potrà verificare a consuntivo se la spesa sarà aumentata, diminuita, restata costante nel tempo, se le Regioni saranno state in grado di garantire i servizi, una migliore qualità dell'assistenza, se vi sarà stato un disavanzo (più o meno sommerso) o un sostanziale pareggio (ottenuto con o senza modifiche delle addizionali regionali e dei ticket). È il caso di far notare che, come appena sopra calcolato, le minori spese previste dalle Leggi di stabilità per il 2015 e 2016 rispetto alle stime previste nel Patto per la Salute per il periodo 2014-16 sono già pari a 7,594 miliardi, quindi i 2,5 miliardi annui di cui ha parlato il Ministro Lorenzin.

Per altro chiarire se si tratti di tagli reali o minori finanziamenti rispetto al trend e a quanto previsto nel Patto per la Salute del 2014 non è un dettaglio, poiché sul piano sostanziale il Ministro Lorenzin si è battuta in sede di Legge di Stabilità, e ha più volte dichiarato di aver ottenuto che «la razionalizzazione e la lotta agli sprechi e alle inefficienze avrebbero determinato risparmi che sarebbero rimasti all'interno del SSN, per potenziare gli investimenti». Ma se il finanziamento totale non è aumentato, o è aumentato di un solo miliardo per il 2016 rispetto al 2015, vuol dire che i risparmi rispetto alle previsioni non sono stati mantenuti all'interno del sistema. Inoltre, a rigor di logica, vuol dire che il SSN non viene messo in grado di far fronte alle nuove esigenze e al crescente fabbisogno determinato dal progresso scientifico, tecnologico e delle metodiche assistenziali. Tutti coloro che conoscono l'SSN e che operano al suo interno sanno che negli ultimi 5-6 anni sono stati molto ridotti gli investimenti per il rinnovo delle attrezzature e apparecchiature «necessarie per seguire l'evoluzione delle buone pratiche assistenziali» (ovviamente non si fa riferimento agli investimenti per attrezzature e apparecchiature inutili o utilizzate per prestazioni inappropriate o collegate a comportamenti di sola medicina difensiva). Investimenti ben al di sotto del livello (percentuale sulla spesa totale) che tutti gli studi internazionali considerano fisiologico per mantenere la qualità dell'assistenza al passo con il progresso delle conoscenze. Alcune indagini stimano il livello degli investimenti del SSN pari a 1/3 o poco più di quello con- 
siderato fisiologico. Inoltre è noto che anche sul piano edilizio di ospedali e di altre strutture pubbliche vi è un notevole ritardo, sia nella costruzione di nuovi ospedali, ambulatori, strutture per Case della Salute o servizi simili adeguati con i nuovi modelli assistenziali, sia con riferimento alle manutenzioni ordinarie e perfino straordinarie.

La mancanza di investimenti ha un effetto di «moltiplicazione negativa» dei costi futuri. Mentre molti parlano degli effetti negativi del debito pubblico «sulle generazioni future», la maggior parte di essi trascurano di considerare gli «effetti negativi sulle generazioni future» in termini di costi aggiuntivi o di riduzione dei livelli assistenziali determinati dai mancati (o ridotti) investimenti attuali. Si tratta di politiche paragonabili a quelle di imprese che, di fronte a difficoltà di bilancio, hanno privilegiato la riduzione dei costi per ricerca e sviluppo o per il rinnovo degli impianti e delle attrezzature, politiche che, come è noto, si sono rivelate disastrose e hanno portato al fallimento di queste imprese, mentre quelle che hanno avuto il coraggio e la forza di investire in queste attività sono uscite dalla crisi poiché sono state in grado di aumentare i ricavi, che nel caso della tutela della salute vuol dire aumentare $i$ «benefici» non solo per la salute ma anche per l'economia.

Le Regioni, da parte loro, non possono trincerarsi dietro il generico richiamo alle «inderogabili esigenze di salute dei cittadini», né sottovalutare gli indirizzi del Governo secondo cui «definire un livello di finanziamento del SSN inferiore alle previsioni contenute nel Patto per la Salute del 2014, ma comunque in costante, seppur lieve, aumento nei prossimi 3-5 anni» è possibile e sostenibile alle seguenti condizioni:

- agire sulle prestazioni non necessarie/inappropriate;

- contenere $i$ costi per acquisto di beni e servizi tramite un accorpamento delle centrali di acquisto (da 32.000 a poche decine per l'intero settore pubblico) e l'applicazione di prezzi di riferimento o prezzi standard;

- ridurre i costi per medicina difensiva, che qualcuno continua a stimare in 10-15 miliardi (dato mai supportato da evidenze);

- ridurre i posti letto (misura per altro già realizzata in termini massicci negli ultimi 10 anni);

- sviluppare modalità di assistenza sul territorio a minor costo;

- rinegoziare contratti che prevedono costi superiori a quelli di riferimento o a quelli standard (misura che darebbe adito come in passato a migliaia di impugnative, poiché appare incostituzionale).

Per quanto riguarda il primo punto, in ottobre è stato predisposto da parte dei «tecnici del Ministero» e di altre otto istituzioni, tra cui Aifa, Ragioneria Generale dello Stato, Banca d'Italia, Consip, MEF un elenco di poco più di 200 prestazioni «a rischio di inappropriatezza» o per le quali sono stati definiti indicatori di «normalità $»$, ossia numero di prestazioni annue per paziente/cittadino. Senza entrare nel merito di questo elenco, che anche molti medici considerano fondato su una buona base di evidenze, la procedura ha lasciato molto perplessi, poiché la dichiarazione secondo cui «erano state coinvolte le società medico-scientifiche» si è rivelata infondata in quanto risulta che siano stati interpellati solo alcuni clinici o esperti a titolo personale e non in rappresentanza delle società scientifiche.

Mentre è difficile contestare che «tecnici del Ministero», Aifa, ISS, abbiano conoscenze di merito su questo tema, $\dot{e}$ più probabile che i tecnici di Ragioneria Genarle dello Stato, Banca d'Italia, MEF e altri organismi responsabili degli equilibri di finanza pubblica si limitino a confronti tra diversi paesi, che spesso però sono viziati da limiti di comparabilità data la grande differenza nei sistemi di tutela della salute, e siano portati a privilegiare interventi di semplici tagli di spesa. La Consip, da parte sua, ha competenze nel definire come comprare al meglio beni, prestazioni e servi$z i$, non certo nel contribuire a definire cosa deve intendersi per appropriato/non appropriato per i pazienti. Non si può che essere d'accordo con un medico che in un articolo pubblicato sul Corriere della Sera l's novembre sottolineava che economisti, manager, esperti di finanza pubblica dovrebbero conoscere a fondo le realtà nelle quali si trovano ad operare medici, infermieri, altro personale di assistenza, prima di «sparare», «proporre», «imporre» soluzioni che non di rado fanno a pugni con la concreta realtà e che hanno il prevalente effetto di demotivare le persone.

Per quanto riguarda il secondo punto è indubbiamente di grande impatto comunicativo sostenere che il Governo si propone di ridurre da 32.000 a poche decine le centrali di acquisto di tutte le amministrazioni del settore pubblico. Su 
questo piano la Consip e le centrali di acquisto generali che già esistono possono dare un contributo di idee e di possibili soluzioni, ma nessuno deve illudersi che questo processo possa avvenire in tempi brevi e nelle dimensioni prospettate. Avere poche centrali di acquisto potrebbe indubbiamente consentire di avere economie di dimensioni nei volumi di acquisto, di specializzazione (per quanto riguarda le procedure e le analisi del mercato dei fornitori) e di forza contrattuale nei confronti dei fornitori. Tuttavia non può sfuggire a nessuno che tali processi richiedano mobilità di personale (che sarà facilitata dalla riforma della PA ma che non sarà comunque semplice), strutture organizzative adeguate, ma che non devono essere trascurati i possibili effetti negativi in termini di tempi lunghi nelle procedure centralizzate, necessità di avere comunque acquisti in deroga per esigenze di urgenza (che nel sistema di tutela della salute non sono banali né rare), standardizzazione delle procedure che possono determinare non una semplificazione ma un ulteriore grado di burocratizzazione. Senza considerare che in presenza di gare per ammontare elevati degli acquisiti può aumentare il rischio di fenomeni di corruzione magari meno numerosi ma per importi più elevati. Anche in questo caso si può citare il detto popolare «piatto ricco mi ci ficco», ben noto ai professionisti della corruzione. La razionalizzazione dei processi di acquisto è una via da perseguire con determinazione e costanza ma con realismo e senza illusione di ottenere risultati eclatanti nel breve periodo. Chi parla di possibili risparmi del 10-15\% in uno o due anni, come è avvenuto e avviene in molte imprese private (affermazioni sentite in alcuni talk show o riportate dai media), dimostra di non conoscere le caratteristiche peculiari del sistema di tutela della salute, dei comportamenti di pazienti oltre che di medici, infermieri e altri operatori, della differenza tra «tutela di un diritto della persona universalmente riconosciuto $e$ 《scegliere autonomamente specifici target di clienti/prodotti $\gg$. In sostanza dimostra di avere una visione «ideologica» del management e della razionalità, non una conoscenza del management, della razionalità, della funzionalità applicata al mondo reale.

Un'altra retorica ricorrente riguarda la necessità e la possibilità di ridurre «gli enormi costi correlati alla medicina difensiva $\gg$. Il problema è reale e rilevante, ma questo obiettivo non può essere conseguito con dichiarazioni, norme inserite in Leggi di Stabilità o indirizzi da parte del Ministero o delle Regioni. Occorre che a livello centrale e a livello regionale siano messe a punto normative in grado di dare una equilibrata risposta a due diverse esigenze che vanno parimenti garantite: quella dei cittadini di essere rispettati in quanto persone e di essere tutelati rispetto a comportamenti di effettiva mal practice e quella dei medici e degli altri operatori di non vivere continuamente sotto la spada di Damocle delle denunce di presunta mal practice che nella stragrande maggioranza dei casi (97-98\% e forse più) si rivelano infondate. La distinzione tra comportamenti dolosi, colpa grave, colpa lieve, situazioni impreviste sono importanti e devono essere normate con equilibrio e affidate a un sistema organico di forme assicurative private, assicurazioni da parte delle aziende per responsabilità oggettiva, assicurazioni regionali. Lasciare singoli medici, infermieri, altri operatori di fronte alla responsabilità personale e all'obbligo di assicurarsi, non può che alimentare e aumentare la medicina difensiva e far diventare lettera morta o grida manzoniane la lotta a questa categoria di spesa.

Tra le misure sono poi previste disincentivi o sanzioni per i medici che prescrivono analisi e controlli superflui, inappropriati, motivati solo da logiche di medicina difensiva. Questo ha suscitato una «levata di scudi $\gg$ che, come sempre in questi casi, deriva da due motivazioni di segno opposto. Alcuni considerano questi eventuali interventi come un «attentato all'autonomia del medico e del professionista» e si oppongono a essi a prescindere dalla necessità oggettiva di evitare spese che non portano benefici per i pazienti. Altri, pur sensibili al problema e sinceramente convinti della necessità di affrontarlo, sottolineano che è inapplicabile o inefficace qualsiasi misura che si limita a scaricare sugli individui (medici, infermieri e altri operatori) la responsabilità per fatti inesistenti (mal practice) o che spesso derivano non da mancanza di personalità e dai propri comportamenti ma da carenze di strutture, attrezzature, personale.

Per tranquillizzare i MMG, i PLS e i medici del SSN, nei giorni successivi all'emanazione della lista delle prestazioni a rischio di inappropriatezza, il Ministro si è affrettato a puntualizzare che «il medico potrà motivare le sue scelte, $e$ che la perdita di incentivi e le sanzioni potranno essere applicate solo previo contraddittorio con l'azienda sanitaria competente». Qualcuno ha pensato a cosa vuol dire concretamente questa affermazione apparentemente tranquillizzante? Le ASL che già hanno difficoltà a garantire le prestazioni ed erogare i servizi previsti dai LEA, dovrebbero 
mettere in campo strumenti per realizzare il contraddittorio con propri medici dipendenti o medici convenzionati quali sono i MMG e i PLS. Qualcuno osa immaginare la perdita di tempo e le possibili conclusioni di tale contraddittorio? È facilmente prevedibile che, se applicata, questa misura produrrà un ulteriore carico burocratico, un aumento di conflitti, o sarà completamente disattesa. Anche in questo caso si può citare un detto popolare secondo cui «il rattoppo è peggio del buco $\gg$ che suonerebbe meglio in dialetto veneto.

Per quanto riguarda la riduzione dei posti letto, tale misura va riferita a un numero assai limitato di Regioni poiché la maggior parte di esse ha già raggiunto lo standard di 3,7 posti letto per mille abitanti. In un numero maggiore di regioni vi è spazio per modificare il mix, riducendo il numero di posti letto per acuti (attualmente superiore al 3 per mille) e aumentare la quota di posti letto per riabilitazione (attualmente inferiore allo 0,7 per mille). Si può aggiungere che con il progresso delle conoscenze e con le nuove metodiche di diagnosi, cura e riabilitazione è addirittura possibile scendere sotto questi standard, ma i risparmi sarebbero comunque contenuti poiché è noto che il driver dei costi da tempo non è più il posto letto, ma l'intensità delle cure, il tipo di tecnologie e di farmaci utilizzati che sono sempre più specifici ma a costi unitari sempre più elevati.

Qualche risparmio può essere sicuramente ottenuto applicando modelli di continuità delle cure tramite il potenziamento dell'assistenza in strutture a bassa intensità di cura (presidi ospedalieri di territorio o ospedali di vicinato) $e$ l'assistenza senza ricovero in case della salute o con altre modalità che in molte regioni sono già state sperimentate con successo. Certamente un contributo in questa direzione potrà essere dato anche dalla diffusione di strumenti di telemedicina e di tecnologie che consentano il monitoraggio a distanza dei pazienti o la possibilità di rapido consulto tra medici di medicina generale e medici specialisti degli ospedali o delle strutture specialistiche di riferimento. Se si vuole garantire una elevata qualità dell'assistenza «che va verso il paziente e i suoi luoghi di residenza» (esempio assisten$z a$ domiciliare), non si può pensare che si possano ottenere elevati risparmi. L'obiettivo che ci si deve porre è quello di migliorare decisamente il rapporto tra qualità dell'assistenza e livello dei costi. Da questa misura non si può sperare di ottenere elevati risparmi a meno di non scaricare parte dei costi sui pazienti e sulle famiglie come oggi purtroppo avviene in parte rilevante per l'assistenza a pazienti cronici e lungodegenti. Una misura di contenimento della spesa del SSN che scaricasse i costi reali dell'assistenza sulle famiglie potrebbe contribuire nel breve periodo alla sostenibilità della finanza pubblica, ma nel medio periodo creerebbe gravi problemi di sostenibilità sociale e, conseguentemente, di sostenibilità e credibilità politica.

In conclusione si può dire che gli obiettivi perseguiti con il combinato disposto della manovra di luglio e della Legge di Stabilità per il 2016 è condizionato a due principali se:

- se si riuscirà a sostituire gli attuali comportamenti con comportamenti più virtuosi, i 111 miliardi saranno sufficienti;

- se i vincoli creati dal razionamento/limitatezza del finanziamento riusciranno a incidere su sprechi, inefficienze, inappropriatezze, corruzione, sarà possibile mantenere livelli quali-quantitativi dei servizi e delle prestazioni erogate ai pazienti e in generale ai cittadini (per gli interventi di prevenzione e di sanità pubblica).

Non è chiaro se la prima condizione debba intendersi più come «un auspicio» o come «una speranza» riposta in chi opera nel sistema che, di fronte alle difficoltà del paese, sarebbe indotto a modificare i comportamenti del passato. A chi propende per l'auspicio si ricorda l'ennesimo detto popolare secondo cui «di buone intenzioni è lastricato l'inferno». Nel caso specifico l'inferno sarebbe costituito da un indebolimento non dichiarato, e forse non voluto, del SSN. A chi propende per la speranza nella capacità di reazione di medici, infermieri, altre figure di assistenza, manager delle aziende sanitarie, degli ospedali, delle case della salute, dirigenti degli Assessorati regionali alla salute, si può ricordare che la speranza va sostenuta con qualche incentivo, almeno morale se non anche materiale. Per sostenere la speranza che le cose cambino, esponenti del Governo, delle Regioni, degli enti locali, dei partiti, nonché opinionisti che speculano su reali o presunti casi di malasanità, dovrebbero almeno astenersi da continui giudizi negativi sul SSN italiano. Non si possono screditare direttamente o indirettamente (con inappropriate generalizzazioni che fanno di 
ogni erba un fascio) coloro che operano nel sistema e al tempo stesso chiedere a essi il passaggio da comportamenti poco virtuosi (di pochi o comunque di una minoranza) a comportamenti virtuosi. Con riguardo alla seconda condizione è appena il caso di richiamare l'attenzione su un «sano realismo». In assenza di forti sistemi di controllo, che è cosa diversa da sistemi di controllo burocratici e dettagliati, è molto probabile che la «scarsità» di risorse si rifletta nella riduzione di prestazioni e servizi erogati a favore di gruppi deboli della popolazione, poiché è noto che le lobby che causano sprechi, inefficienze, inappropriatezze, corruzioni sanno difendere molto bene i propri interessi (illeciti). Seguendo il richiamo fatto dal Ministro Padoan secondo cui la Legge di Stabilità va valutata nella sua logica complessiva e non per i singoli provvedimenti, si può cercare di interpretare la politica e la strategia del Governo che possono essere sintetizzate nei seguenti termini: sfruttando la ripresa economica (favorita dal basso prezzo del petrolio e di altre commodities e del quantitative easing della $B C E$ ) e utilizzando i margini di flessibilità consentiti dalla UE, si è preferito disinnescare le clausole di salvaguardia che prevedevano l'aumento automatica dell'IVA in caso di mancato raggiungimento degli obiettivi di finanza pubblica prefissati, ridurre le imposte sulla prima casa, riproporre gli incentivi previsti dal Jobs Act, intervenire a favore di individui e famiglie con basso reddito al fine di aumentare $i$ consumi interni e consolidare la ripresa, ridurre la disoccupazione. Gli oppositori politici o i maligni sostengono che quest'impostazione della manovra di finanza pubblica è finalizzata ad allargare la base del consenso in vista del turno delle elezioni amministrative di primavera 2016. Coloro che sostengono il Governo o sono disposti a dargli credito sperano che il consolidamento della ripresa crei lo spazio economico per aumentare in modo più significativo, a partire dal 2017, il finanziamento al SSN. Ciò vorrebbe dire un «anticipo del futuro».

Mi sento di porre all'attenzione del Primo Ministro Renzi, del Ministro Lorenzin, degli altri Ministri del Governo e di chi sostiene in modo convinto questa strategia, l'esigenza o l'opportunità di «non scherzare con il fuoco». Infatti, se per sostenere questa strategia si continua ad attribuire alle Regioni, alle ASL, agli enti locali, la responsabilità per eventuali riduzioni dei servizi (di tutela della salute e altri erogati dagli enti locali), si finisce per delegittimare più di quanto già non avvenga il ruolo della politica e per alimentare o far soffiare ancor più forte il vento dell'anti-politica (questo è il fuoco) che finirebbe per bruciare il ruolo delle istituzioni con danno per tutti ma soprattutto per $i$ più deboli. Mi permetto di ricordare che sostenibilità economica (della finanza pubblica), sostenibilità politica e sostenibilità sociale sono tre dimensioni che non possono essere messe in sequenza ma che devono essere affrontate contestualmente perché interagiscono tra loro. 